PROCEEDINGS OF THE

AMERICAN MATHEMATICAL SOCIETY

Volume 127, Number 6, Pages 1575-1582

S 0002-9939(99)04679-1

Article electronically published on February 5, 1999

\title{
EXISTENCE OF UNLIFTABLE MODULES
}

\author{
DAVID A. JORGENSEN
}

(Communicated by Wolmer V. Vasconcelos)

\begin{abstract}
Let $(Q, \mathfrak{n})$ be a commutative Noetherian local ring, and let $R=$ $Q /(x)$ where $x$ is a non-zerodivisor of $Q$ contained in $\mathfrak{n}$. Then a finitely generated $R$-module $M$ is said to lift to $Q$ if there exists a finitely generated $Q$-module $M^{\prime}$ such that $x$ is $M^{\prime}$-regular and $M \cong M^{\prime} / x M^{\prime}$. In this paper we give a general construction of finitely generated $R$-modules of finite projective dimension over $R$ which fail to lift to $Q$ provided $x \in \mathfrak{n}^{2}$ and the depth of $R$ is at least 2 .
\end{abstract}

\section{INTRODUCTION}

Let $Q$ be a commutative Noetherian local ring with maximal ideal $\mathfrak{n}$, and let $R$ be the quotient of $Q$ by a proper ideal $I$. Suppose that $M$ is a finitely generated $R$-module. To say that $M$ lifts to $Q$ means that there exists a finitely generated $Q$-module $M^{\prime}$ such that $\operatorname{Tor}_{i}^{Q}\left(M^{\prime}, R\right)=0$ for all $i>0$ and $M^{\prime} / I M^{\prime} \cong M$. One may interpret these conditions as saying that the homological features of $M^{\prime}$ as a module over $Q$ are essentially the same as the homological features of $M$ as a module over $R$. For instance, if $N$ is any $R$-module, there are natural isomorphisms $\operatorname{Tor}_{i}^{R}(M, N) \cong \operatorname{Tor}_{i}^{Q}\left(M^{\prime}, N\right)$ and $\operatorname{Ext}_{R}^{i}(M, N) \cong \operatorname{Ext}_{Q}^{i}\left(M^{\prime}, N\right)$ for all $i$.

The motivation for studying lifting is that, in most applications, homological algebra of modules over $Q$ is better understood than homological algebra of modules over $R$. Hence information involving the homological characteristics of a module over $R$ is made more accessible upon knowing the module lifts to $Q$.

While it is desirable to establish sufficient conditions for the ability to lift $R$ modules to $Q$, the purpose of this paper is to show that quite often there are $R$-modules which fail to lift to $Q$. Hochster $[\mathrm{H}]$ and Hoffman [Ho] have shown that there exist complete regular local rings $Q$ of mixed characteristic, elements $x \in \mathfrak{n}-\mathfrak{n}^{2}$, and, for $R=Q /(x)$, cyclic $R$-modules $R / \mathfrak{a}$, with $\mathfrak{a}$ generated by at least 8 elements, which fail to lift to $Q$. (These were given as counterexamples to "Grothendieck's lifting problem".) Peskine and Szpiro have shown [PS] that there exist finitely generated - but not necessarily cyclic $-R$-modules of finite projective dimension over $R$ which fail to lift to $Q$ whenever $Q$ is a regular local ring and $I$ is a principal ideal generated by $x$ in $\mathfrak{n}^{2}$. We show in Section 2 that there exist both non-cyclic (2.3) and cyclic (2.4) $R$-modules of finite projective dimension which do not lift to $Q$ in the situation where $(Q, \mathfrak{n})$ is an arbitrary commutative

Received by the editors June 10, 1997 and, in revised form, September 4, 1997.

1991 Mathematics Subject Classification. Primary 13D25, 13 H99.

Key words and phrases. Lifting of modules, free resolution, lifting of complexes, dualized complex. 
Noetherian local ring (of sufficiently high depth) and $I$ is generated by a $Q$-regular sequence with at least one generator contained in $\mathfrak{n}^{2}$. It should be noted that the obstruction to lifting used in our constructions is different from the obstructions used by Hochster, Hoffman and Peskine-Szpiro.

Suppose now that $I$ is a principal ideal of $Q$ generated by a non-zerodivisor. Buchsbaum and Eisenbud have shown [BE1, (9.3)] that their structure theorem for finite free resolutions $[\mathrm{BE} 1,(3.1)]$ provides sufficient information to guarantee that all cyclic $R$-modules $R / \mathfrak{a}$ of projective dimension 3 over $R$, such that $\mathfrak{a}$ is generated by 3 elements, lift to $Q$. Section 3 of this paper consists of two examples, (3.2) and (3.3), which show that this lifting theorem of Buchsbaum and Eisenbud is best possible, in the sense that neither the projective dimension of $R / \mathfrak{a}$, nor the minimal number of generators of $\mathfrak{a}$ can in general be increased in the hypotheses of their lifting theorem.

Section 1 below consists of definitions and an important lemma (1.1) which is used heavily in the sequel.

\section{Preliminaries}

Throughout this paper $Q$ will denote a commutative Noetherian local ring with maximal ideal $\mathfrak{n}$ and residue field $k$, and $R$ will always denote the quotient of $Q$ by a proper ideal $I$. We will assume all $R$-modules are finitely generated.

In this situation, we say that an $R$-module $M$ lifts to $Q$ if there exists a finitely generated $Q$-module $M^{\prime}$ such that

(1) $\operatorname{Tor}_{i}^{Q}\left(M^{\prime}, R\right)=0$ for all $i>0$, and

(2) $M^{\prime} / I M^{\prime} \cong M$.

If such an $M^{\prime}$ exists, we say it is a lifting of $M$ to $Q$.

Let

$$
(\mathbf{F}, \partial): \quad \cdots \longrightarrow F_{i+1} \stackrel{\partial_{i+1}}{\longrightarrow} F_{i} \stackrel{\partial_{i}}{\longrightarrow} F_{i-1} \stackrel{\partial_{i-1}}{\longrightarrow} \cdots
$$

be a complex of free $R$-modules. We say that the complex $\mathbf{F}$ lifts to $Q$ if there exists a sequence of free $Q$-modules with maps between them

$$
(\mathbf{L}, d): \quad \cdots \longrightarrow L_{i+1} \stackrel{d_{i+1}}{\longrightarrow} L_{i} \stackrel{d_{i}}{\longrightarrow} L_{i-1} \stackrel{d_{i-1}}{\longrightarrow} \cdots
$$

such that

(1) $(\mathbf{L}, d)$ is a complex, and

(2) $\mathbf{L} \otimes_{Q} R$ and $\mathbf{F}$ are isomorphic as complexes.

If such a sequence $(\mathbf{L}, d)$ exists, we say it is a lifting of $\mathbf{F}$ to $Q$.

Let $M$ be an $R$-module. Recall that a complex of free $R$-modules $(\mathbf{F}, \partial)$ is an $R$-free resolution of $M$ if $F_{i}=0$ for all $i<0, \mathbf{F}$ is acyclic (which means $\mathrm{H}_{i}(\mathbf{F})=0$ for all $i>0$ ), and $\mathrm{H}_{0}(\mathbf{F}) \cong M$.

As the reader may suspect, there is a close relationship between the ability to lift $R$-modules and the ability to lift their resolutions. The following is a strengthening of [BE1, (9.1)].

(1.1) Lemma. Let $M$ be an R-module. Then the following are equivalent:

(1) $M$ lifts to $Q$.

(2) Every $R$-free resolution of $M$ has an acyclic lifting to $Q$.

(3) There exists an $R$-free resolution $(\mathbf{F}, \partial)$ of $M$ with some lifting to $Q$ which is acyclic. 
Whenever I is a principal ideal generated by a non-zerodivisor of $Q$, (3) above may be replaced by

$\left(3^{\prime}\right)$ There exists an $R$-free resolution $(\mathbf{F}, \partial)$ of $M$ such that the complex $0 \rightarrow$ $F_{2} \stackrel{\partial_{2}}{\longrightarrow} F_{1} \stackrel{\partial_{1}}{\longrightarrow} F_{0} \rightarrow 0$ has some lifting to $Q$.

Proof. $(2) \Rightarrow(3),\left(3^{\prime}\right)$ are trivial.

$(3) \Rightarrow(1)$ : Let $(\mathbf{L}, d)$ be an acyclic lifting of $(\mathbf{F}, \partial)$. Then $\mathbf{L}$ is a $Q$-free resolution of the $Q$-module $M^{\prime}:=$ coker $d_{1}$. Since $\mathbf{L} \otimes_{Q} R \cong \mathbf{F}$, we have $\operatorname{Tor}_{i}^{Q}\left(M^{\prime}, R\right) \cong$ $\mathrm{H}_{i}(\mathbf{F})=0$ for all $i>0$. Also $M^{\prime} / I M^{\prime} \cong M^{\prime} \otimes_{Q} R \cong M$. Hence $M^{\prime}$ is a lifting of $M$.

$\left(3^{\prime}\right) \Rightarrow(1)$ : Write $I=(x)$ where $x$ is a non-zerodivisor of $Q$. Suppose $(\mathbf{L}, d)$ is a lifting of $0 \rightarrow F_{2} \stackrel{\partial_{2}}{\longrightarrow} F_{1} \stackrel{\partial_{1}}{\longrightarrow} F_{0} \rightarrow 0$. It is an easy application of Nakayama's lemma to show that $\mathbf{L}$ is then actually exact at $L_{1}$. This implies that $\operatorname{Tor}_{1}^{Q}\left(M^{\prime}, R\right)=0$, where $M^{\prime}:=\operatorname{coker} d_{1} . \operatorname{As}_{\operatorname{pd}_{Q}} R=1$, we actually have that $\operatorname{Tor}_{i}^{Q}\left(M^{\prime}, R\right)=0$ for all $i>0$, as desired.

$(1) \Rightarrow(2)$ : Let $M^{\prime}$ be a lifting of $M$, and let $(\mathbf{L}, d)$ be any minimal $Q$-free resolution of $M^{\prime}$. Then the two conditions in the definition of lifting of an $R$ module guarantee that $\left(\mathbf{L} \otimes_{Q} R, d \otimes_{Q} R\right)$ is a minimal $R$-free resolution of $M$. Since any two minimal resolutions of the same module (over the same local ring) are isomorphic as complexes, we conclude that every minimal free resolution of $M$ lifts to $Q$.

Now let $\mathbf{F}$ be an arbitrary $R$-free resolution of $M$. It is well-known that $\mathbf{F}$ is isomorphic to a complex of the form

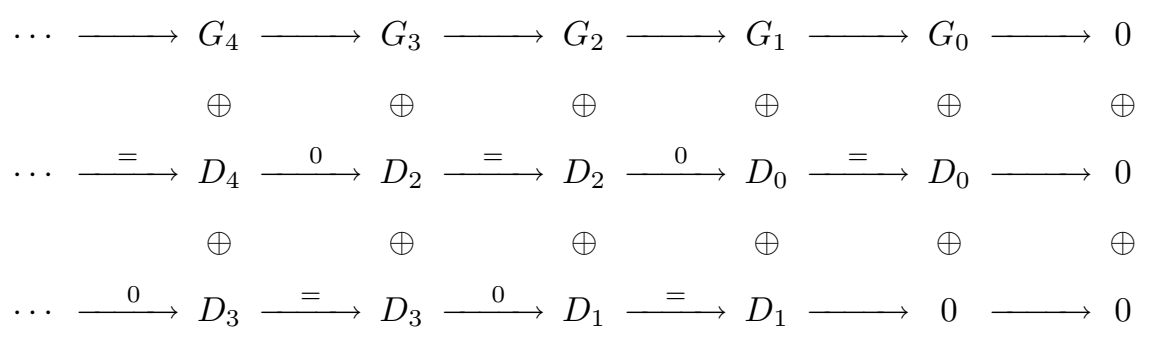

where $\mathbf{G}$ is a minimal $R$-free resolution of $M$, and the $D_{i}$ are free $R$-modules. Since $\mathbf{G}$ lifts to $Q$, so does the complex above. Hence $\mathbf{F}$ lifts to $Q$.

(1.2) Remark. Suppose $I$ is generated by a $Q$-regular sequence $x_{1}, \ldots, x_{c}$, and let $M$ be an $R$-module. Then it's easy to see that the existence of a lifting $M^{\prime}$ of $M$ to $Q$ implies that there exist modules $M_{1}, \ldots, M_{c}=M^{\prime}$ such that $M_{1}$ is a lifting of $M$ to $Q /\left(x_{1}, \ldots, x_{c-1}\right), M_{2}$ is a lifting of $M_{1}$ to $Q /\left(x_{1}, \ldots, x_{c-2}\right)$, and so on. Hence lifting mod a regular sequence is equivalent to lifting mod a non-zerodivisor at each stage. It follows that (1) and $\left(3^{\prime}\right)$ of $(1.1)$ are equivalent when $I$ is generated by a $Q$-regular sequence of arbitrary length.

Remark. The following example illustrates that the hypothesis " $I$ is generated by a non-zerodivisor" is essential for the equivalence of $(1)$ and $\left(3^{\prime}\right)$ above: Let $Q:=$ $k[[X, Y]] /(X Y)$ (for $k$ a field) and $I:=(x)$. Then a free $R$-resolution of $R /(\bar{y})$ (where ${ }^{-}$denotes elements of $Q \bmod x$ ) is given by $0 \rightarrow R \stackrel{\bar{y}}{\longrightarrow} R \rightarrow 0$. This resolution has the obvious lifting $0 \rightarrow Q \stackrel{y}{\longrightarrow} Q \rightarrow 0$ to $Q$. However, this lifting is not acyclic. 


\section{The EXIStence}

In this section we establish the existence of unliftable modules in the situation where some member of a minimal set of generators of $I$ is contained in the square of the maximal ideal of $Q$.

(2.1) Lemma. Let $x_{1}, \ldots, x_{c}$ be a minimal generating set for the ideal I. Suppose $K$ is a proper ideal of $Q$ containing $I$ such that $x_{1} \in \mathfrak{n} K$. Then for each ideal $J$ of $Q, I+J=K$ implies $I J \neq I \cap J$.

Proof. Let $m_{1}, \ldots, m_{e}$ be a minimal generating set of $K$. Write

$$
x_{1}=r_{1} m_{1}+\cdots+r_{e} m_{e}
$$

with $r_{i} \in \mathfrak{n}$ for all $i$.

Suppose that $J$ is an ideal of $Q$ with $I+J=K$. For each $i, 1 \leq i \leq e$, write $m_{i}=m_{i}^{\prime}+m_{i}^{\prime \prime}$, where $m_{i}^{\prime} \in I$ and $m_{i}^{\prime \prime} \in J$. After substituting these expressions for the $m_{i}$ into (2.1.1) and rearranging, we see that

$$
x_{1}-r_{1} m_{1}^{\prime}-\cdots-r_{e} m_{e}^{\prime}=r_{1} m_{1}^{\prime \prime}+\cdots+r_{e} m_{e}^{\prime \prime} \in I \cap J .
$$

Since $\sum r_{i} m_{i}^{\prime} \in \mathfrak{n} I$, the element $x_{1}-r_{1} m_{1}^{\prime}-\cdots-r_{e} m_{e}^{\prime}$ is a member of a minimal generating set of $I$, and therefore cannot belong to the ideal $I J$. Hence $I J \neq$ $I \cap J$.

(2.2) Theorem. Let $x_{1}, \ldots, x_{c}$ be a minimal generating set for the ideal $I$, and suppose that $x_{1} \in \mathfrak{n}^{2}$. If $K$ is a proper ideal of $Q$ containing $I$ such that $x_{1} \in \mathfrak{n} K$, then the $R$-module $Q / K$ does not lift to $Q$. In particular, the residue field $k$ does not lift to $Q$.

Proof. It is clear that lifting $Q / K$ to $Q$ is equivalent to finding an ideal $J$ of $Q$ satisfying

(1) $\operatorname{Tor}_{i}^{Q}(Q / J, Q / I)=0$ for all $i>0$, and

(2) $I+J=K$.

Since $\operatorname{Tor}_{1}^{Q}(Q / J, Q / I) \cong \frac{I \cap J}{I J},(2.1)$ shows that no ideal $J \subseteq Q$ satisfies properties (1) and (2) simultaneously.

Such modules $Q / K$ as in (2.2) are likely to have infinite projective dimension over $R$. Since in many cases (for instance, when $Q$ is regular but $R$ is not) infinite projective dimension is itself an obstruction to lifting, and since many important homological questions involve modules of finite projective dimension, it is of interest to establish the existence of $R$-modules of finite projective dimension over $R$ which fail to lift to $Q$. To achieve this, we will restrict our situation to where $I$ is generated by a $Q$-regular sequence, and then use (2.2).

For an $R$-module $M$, we let $M^{*}$ denote the $R$-module $\operatorname{Hom}_{R}(M, R)$; for a $Q$ module $N$, we let $N^{\vee}$ denote the $Q$-module $\operatorname{Hom}_{Q}(N, Q)$. When $M$ is non-zero, we let $\operatorname{grade}_{R} M$ denote the non-negative integer $\min \left\{i \mid \operatorname{Ext}_{R}^{i}(M, R) \neq 0\right\}$. (See, e.g., $[\mathrm{BH},(1.2 .11)]$.)

(2.3) Theorem. Suppose that $I$ is generated by a $Q$-regular sequence $x_{1}, \ldots, x_{c}$ such that $x_{1} \in \mathfrak{n}^{2}$. Assume that the depth $d$ of $R$ is at least two. Let $j$ be an integer with $2 \leq j \leq d$. Then there exist $R$-modules of projective dimension $j$ over $R$ which fail to lift to $Q$. 
Proof. Let $A$ be an $R$-module with grade ${ }_{R} A \geq j$ which does not lift to $Q$. (For example, one could take $A=k$.) Let $(\mathbf{F}, \partial)$ be an $R$-free resolution of $A$. Since $\operatorname{Ext}_{R}^{i}(A, R)=0$ for all $i, 0 \leq i<j$, the dualized complex

$$
\mathbf{G}(A, j): 0 \longrightarrow F_{0}^{*} \stackrel{\partial_{1}^{*}}{\longrightarrow} F_{1}^{*} \stackrel{\partial_{2}^{*}}{\longrightarrow} \cdots \stackrel{\partial_{j}^{*}}{\longrightarrow} F_{j}^{*} \longrightarrow 0
$$

is acyclic. Hence $M(A, j):=$ coker $\partial_{j}^{*}$ is an $R$-module of projective dimension $j$ over $R$. We claim that $M(A, j)$ fails to lift to $Q$.

Suppose that $M(A, j)$ does lift to $Q$. By $(1.1)$, there exists a lifting $(\mathbf{L}, d)$ of $\mathbf{G}(A, j)$ to $Q$. It follows that $\left(\mathbf{L}^{\vee}, d^{\vee}\right)$ is a lifting to $Q$ of the complex $0 \rightarrow F_{j} \stackrel{\partial_{j}}{\longrightarrow}$ $\cdots \stackrel{\partial_{2}}{\longrightarrow} F_{1} \stackrel{\partial_{1}}{\longrightarrow} F_{0} \rightarrow 0$. Hence the complex $0 \rightarrow L_{j-2}^{\vee} \stackrel{d_{j-1}^{\vee}}{\longrightarrow} L_{j-1}^{\vee} \stackrel{d_{j}^{\vee}}{\longrightarrow} L_{j}^{\vee} \rightarrow 0$ is a lifting to $Q$ of the complex $0 \rightarrow F_{2} \stackrel{\partial_{2}}{\longrightarrow} F_{1} \stackrel{\partial_{1}}{\longrightarrow} F_{0} \rightarrow 0$. But now (1.2) says that $A$ lifts to $Q$, which is a contradiction.

The module $M(A, j)$ whose existence is asserted in (2.3) is most often not cyclic. However, we can produce examples of cyclic modules which fail to lift to $Q$ if the depth of $R$ is large enough:

(2.4) Theorem. Suppose that $I$ is generated by a $Q$-regular sequence $x_{1}, \ldots, x_{c}$ such that $x_{1} \in \mathfrak{n}^{2}$. Assume that the depth of $R$ is at least four. Let $A$ be an $R$-module with grade $_{R} A \geq 4$ which does not lift to $Q$. (For example, one could take $A=k$.) Fix integers $j$ and $m$ such that $4 \leq j \leq \operatorname{grade}_{R} A$ and $1 \leq m \leq \operatorname{grade}_{R} A-j+1$. Then there exists a cyclic $R$-module $R / \mathfrak{a}(A, j, m)$ of projective dimension $j+m-1$ over $R$ which fails to lift to $Q$.

Proof. Let $(\mathbf{F}, \partial)$ be an $R$-free resolution of $A$. Since $\operatorname{Ext}_{R}^{i}(A, R)=0$ for all $i$, $0 \leq i<j$, the dualized complex

$$
\mathbf{G}(A, j): 0 \longrightarrow F_{0}^{*} \stackrel{\partial_{1}^{*}}{\longrightarrow} F_{1}^{*} \stackrel{\partial_{2}^{*}}{\longrightarrow} \cdots \stackrel{\partial_{j}^{*}}{\longrightarrow} F_{j}^{*} \longrightarrow 0
$$

is acyclic. Set $C(A, j):=$ image $\partial_{j}^{*}$. Then $C(A, j)$ is an $m^{\text {th }}$ syzygy module of finite projective dimension over $R$. Let $r:=\operatorname{rank}\left(\right.$ image $\left.\partial_{j-1}^{*}\right)$. (Every $R$-module of finite projective dimension over $R$ has a rank [BH, (1.4.5)].) Now Satz 3 of [B] establishes the existence of an acyclic complex

$$
\begin{aligned}
& \mathbf{E}(A, j, m): \quad 0 \rightarrow F_{0}^{*} \stackrel{\partial_{1}^{*}}{\longrightarrow} F_{1}^{*} \stackrel{\partial_{2}^{*}}{\longrightarrow} \cdots \stackrel{\partial_{j-2}^{*}}{\longrightarrow} F_{j-2}^{*} \stackrel{f^{\prime}}{\longrightarrow} R^{r+m} \stackrel{f_{m}}{\longrightarrow} \\
& R^{2 m-1} \stackrel{f_{m-1}}{\longrightarrow} R^{2 m-3} \stackrel{f_{m-2}}{\longrightarrow} \cdots \stackrel{f_{2}}{\longrightarrow} R^{3} \stackrel{f_{1}}{\longrightarrow} R \rightarrow 0 .
\end{aligned}
$$

Let $\mathfrak{a}(A, j, m):=$ image $f_{1}$. We claim that $R / \mathfrak{a}(A, j, m)$ does not lift to $Q$.

Suppose that $R / \mathfrak{a}(A, j, m)$ does lift to $Q$. Then, by (1.1), there exists a lifting $(\mathbf{L}, d)$ to $Q$ of $\mathbf{E}(A, j, m)$. It follows that $\left(\mathbf{L}^{\vee}, d^{\vee}\right)$ is a lifting to $Q$ of the complex

$$
\begin{aligned}
& 0 \rightarrow R^{*} \stackrel{f_{1}^{*}}{\longrightarrow} R^{3^{*}} \stackrel{f_{2}^{*}}{\longrightarrow} \cdots \stackrel{f_{m-2}^{*}}{\longrightarrow} R^{2 m-3^{*}} \stackrel{f_{m-1}^{*}}{\longrightarrow} R^{2 m-1^{*}} \stackrel{f_{m}^{*}}{\longrightarrow} \\
& R^{r+m^{*}} \stackrel{f^{\prime *}}{\longrightarrow} F_{j-2} \stackrel{\partial_{j-2}}{\longrightarrow} \cdots \stackrel{\partial_{2}}{\longrightarrow} F_{1} \stackrel{\partial_{1}}{\longrightarrow} F_{0} \rightarrow 0 .
\end{aligned}
$$

This in turn means that the complex $0 \rightarrow L_{j+m-3}^{\vee} \stackrel{d_{j+m-2}^{\vee}}{\longrightarrow} L_{j+m-2}^{\vee} \stackrel{d_{j+m-1}^{\vee}}{\longrightarrow} L_{j+m-1}^{\vee}$ $\rightarrow 0$ is a lifting to $Q$ of the complex $0 \rightarrow F_{2} \stackrel{\partial_{2}}{\longrightarrow} F_{1} \stackrel{\partial_{1}}{\longrightarrow} F_{0} \rightarrow 0$. Now (1.2) says that $A$ lifts to $Q$, which is a contradiction. 
Remark. Note that if $m \geq 2$ in the hypotheses of (2.4) (which forces depth $R \geq 5$ ), then $\mathfrak{a}(A, j, m)$ is generated by 3 elements. It is known that if $\mathfrak{a}$ is an ideal of $R$ generated by $\leq 2$ elements and $R / \mathfrak{a}$ has finite projective dimension over $R$, then $\operatorname{pd}_{R} R / \mathfrak{a} \leq 2$ [BE2, Theorem 2]. If the projective dimension over $R$ of a cyclic $R$-module $R / \mathfrak{a}$ is 2 , the Hilbert-Burch Theorem implies that $R / \mathfrak{a}$ lifts to $Q$. (See [BE1, (9.2)].) If $\operatorname{pd}_{R} R / \mathfrak{a}=1$ then (a is principal and) $R / \mathfrak{a}$ lifts to $Q$ trivially by $(1.2)$.

\section{EXAMPLES}

Assume that $I$ is a principal ideal generated by a non-zerodivisor $x$. The lifting theorem of Buchsbaum and Eisenbud [BE1, 9.3] states that every cyclic $R$-module $R / \mathfrak{a}$, such that $\operatorname{pd}_{R} R / \mathfrak{a}=3$ and $\mathfrak{a}$ is generated by 3 elements, lifts to $Q$. (Their lifting theorem holds even when $x \in \mathfrak{n}-\mathfrak{n}^{2}$.) The purpose of this section is to present two examples which show, in the case where $x \in \mathfrak{n}^{2}$, that this lifting theorem of Buchsbaum and Eisenbud cannot in general be extended to cyclic modules defined by an ideal needing 3 generators and of projective dimension greater than 3 , nor to projective dimension 3 cyclic modules defined by an ideal needing more than 3 generators. The method used to obtain these examples is similar to the method of (2.4). However, we need a lemma, a more technical version of (1.1), (which holds for any proper ideal $I$ of $Q$ ) in order to prove that these examples do not lift. We state this lemma now and defer its proof to the end of the section.

If $B$ is a matrix with entries in $R$, we shall call a matrix $A$ with entries in $Q$ a lifting of $B$ to $Q$ if the $(i, j)$-th entry of the matrix $A$ is a pre-image of the $(i, j)$-th entry of the matrix $B$, for all $i$ and $j$.

(3.1) Lemma. Let $M$ be an R-module which lifts to $Q$. Let

$$
(\mathbf{F}, \partial): \quad \cdots \rightarrow R^{b_{i+1}} \stackrel{\partial_{i+1}}{\longrightarrow} R^{b_{i}} \stackrel{\partial_{i}}{\longrightarrow} \cdots \stackrel{\partial_{2}}{\longrightarrow} R^{b_{1}} \stackrel{\partial_{1}}{\longrightarrow} R^{b_{0}} \rightarrow 0
$$

be an $R$-free resolution of $M$, where the maps $\partial_{i}$ are represented by matrices with respect to the standard bases of the $R^{b_{i}}$. Then there exist liftings $\tilde{\partial}_{i}$ to $Q$ of the matrices $\partial_{i}$ such that the resulting sequence

$$
(\widetilde{\mathbf{F}}, \tilde{\partial}): \quad \cdots \rightarrow Q^{b_{i+1}} \stackrel{\tilde{\partial}_{i+1}}{\longrightarrow} Q^{b_{i}} \stackrel{\tilde{\partial}_{i}}{\longrightarrow} \cdots \stackrel{\tilde{\partial}_{2}}{\longrightarrow} Q^{b_{1}} \stackrel{\tilde{\partial}_{1}}{\longrightarrow} Q^{b_{0}} \rightarrow 0
$$

is an acyclic complex.

(3.2) Example. Let $k$ be a field, let $Q:=k\left[\left[X_{1}, X_{2}, X_{3}, X_{4}, X_{5}\right]\right]$, and let $R:=$ $Q /\left(X_{1} X_{2}-X_{3}^{2}\right)$. Set $\mathfrak{a}:=\left(a_{1}, a_{2}, a_{3}\right)$, where

$$
\begin{aligned}
& a_{1}:=x_{1}^{2} x_{3}-x_{2} x_{3}^{2}+x_{3}^{2} x_{4}-x_{2} x_{4}^{2}+x_{2} x_{3} x_{5}+x_{1} x_{4} x_{5}+x_{5}^{3}, \\
& a_{2}:=-x_{1} x_{3}^{2}-x_{3}^{3}+x_{2} x_{3} x_{4}-x_{1} x_{4}^{2}-x_{2}^{2} x_{5}+x_{1} x_{3} x_{5}-x_{4} x_{5}^{2}, \\
& a_{3}:=x_{3}^{2} x_{4}-x_{2} x_{3} x_{5}+x_{2} x_{5}^{2} .
\end{aligned}
$$

Then $\operatorname{pd}_{R} R / \mathfrak{a}=4$ and $R / \mathfrak{a}$ does not lift to $Q$.

Proof. It can be shown (say, with MACAULAY [Mac]) that there is a minimal free resolution of $R / \mathfrak{a}$ of the form

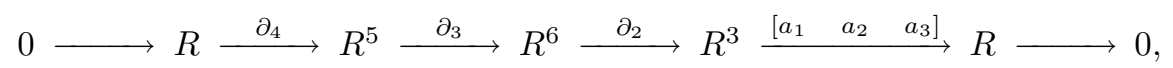


where, with respect to the standard bases of $R, R^{5}$ and $R^{6}$, the matrices

$$
\left[\begin{array}{c}
x_{1} \\
-x_{5} \\
x_{4} \\
-x_{3} \\
x_{2}
\end{array}\right] \quad \text { and } \quad\left[\begin{array}{ccccc}
x_{2} & -x_{3} & 0 & x_{5} & -x_{1} \\
x_{4} & 0 & -x_{1} & -x_{2} & -x_{3} \\
0 & x_{2} & x_{3} & x_{4} & x_{5} \\
-x_{5} & -x_{1} & -x_{2} & 0 & x_{4} \\
-x_{3} & x_{4} & x_{5} & -x_{1} & 0 \\
x_{2} & 0 & 0 & x_{3} & 0
\end{array}\right]
$$

represent the maps $\partial_{4}$ and $\partial_{3}$, respectively.

Suppose that $R / \mathfrak{a}$ lifts to $Q$. Then, by (3.1), there exist liftings $\tilde{\partial}_{4}$ and $\tilde{\partial}_{3}$ to $Q$ of the matrices $\partial_{4}$ and $\partial_{3}$, respectively, such that the product matrix $\tilde{\partial}_{3} \tilde{\partial}_{4}$ is the zero matrix. Write

$$
\tilde{\partial}_{4}=\left[\begin{array}{c}
X_{1}+r_{1} f \\
-X_{5}+r_{2} f \\
X_{4}+r_{3} f \\
-X_{3}+r_{4} f \\
X_{2}+r_{5} f
\end{array}\right], \quad \text { and } \quad\left[\begin{array}{lllll}
X_{2}+s_{1} f & 0+s_{2} f & 0+s_{3} f & X_{3}+s_{4} f & 0+s_{5} f
\end{array}\right]
$$

for the $6^{\text {th }}$ row of $\tilde{\partial}_{3}$, where the $r_{i}$ and $s_{i}$ are in $Q$ and $f:=X_{1} X_{2}-X_{3}^{2}$. We see then that the $(6,1)$-entry in the product matrix $\tilde{\partial}_{3} \tilde{\partial}_{4}$, after expanding and regrouping, has the form $f+r f$, where $r \in \mathfrak{n}$. As this element is clearly non-zero in $Q$, we have reached a contradiction. Hence $R / \mathfrak{a}$ does not lift to $Q$.

(3.3) Example. Let $k$ be a field, let $Q:=k\left[\left[X_{1}, X_{2}, X_{3}, X_{4}\right]\right]$, and let $R:=$ $Q /\left(X_{1} X_{2}-X_{3}^{2}\right)$. Set $\mathfrak{b}:=\left(b_{1}, b_{2}, b_{3}, b_{4}\right)$, where

$$
\begin{aligned}
& b_{1}:=-x_{2} x_{3}+x_{2} x_{4}, \quad b_{2}:=x_{1} x_{3}+x_{2} x_{3}, \\
& b_{3}:=-x_{2}^{2}-x_{3} x_{4}, \quad b_{4}:=x_{1}^{2}-x_{2}^{2}+x_{3}^{2}-x_{4}^{2} .
\end{aligned}
$$

Then $\operatorname{pd}_{R} R / \mathfrak{b}=3$ and $R / \mathfrak{b}$ does not lift to $Q$.

Proof. The proof is similar to the proof of (3.2): It can be shown that $R / \mathfrak{b}$ has a minimal $R$-free resolution of the form

$$
0 \longrightarrow R \stackrel{\partial_{3}}{\longrightarrow} R^{4} \stackrel{\partial_{2}}{\longrightarrow} R^{4} \stackrel{\left[\begin{array}{llll}
b_{1} & b_{2} & b_{3} & b_{4}
\end{array}\right]}{\longrightarrow} R \longrightarrow 0
$$

where, with respect to the standard bases of $R$ and $R^{4}, \partial_{3}$ and $\partial_{2}$ are represented by the matrices

$$
\left[\begin{array}{c}
-x_{1} \\
-x_{2} \\
x_{4} \\
x_{3}
\end{array}\right] \quad \text { and } \quad\left[\begin{array}{cccc}
x_{4} & -x_{3} & x_{1} & -x_{2} \\
-x_{3} & x_{4} & x_{2} & -x_{1} \\
-x_{2} & x_{1} & x_{3} & -x_{4} \\
x_{2} & 0 & 0 & x_{3}
\end{array}\right]
$$

respectively.

Let $\tilde{\partial}_{3}$ and $\tilde{\partial}_{2}$ be liftings to $Q$ of the matrices representing $\partial_{3}$ and $\partial_{2}$, respectively. Write

$$
\tilde{\partial}_{3}=\left[\begin{array}{c}
-X_{1}+r_{1} f \\
-X_{2}+r_{2} f \\
X_{4}+r_{3} f \\
X_{3}+r_{4} f
\end{array}\right] \text { and }\left[\begin{array}{llll}
X_{2}+s_{1} f & 0+s_{2} f & 0+s_{3} f & X_{3}+s_{4} f
\end{array}\right]
$$


for the $4^{t h}$ row of $\tilde{\partial}_{2}$, where the $r_{i}$ and $s_{i}$ are in $Q$ and $f:=X_{1} X_{2}-X_{3}^{2}$. Then the $(4,1)$-entry of the product matrix $\tilde{\partial}_{2} \tilde{\partial}_{3}$ has the form $-f+r f$, where $r \in \mathfrak{n}$. As this element is non-zero in $Q$, there cannot exist a lifting to $Q$ of the $R$-module $R / \mathfrak{b}$.

Remark. There is nothing special about the element $X_{1} X_{2}-X_{3}^{2}$ in (3.2) and (3.3). Undoubtedly one can produce similar examples for any polynomial ring mod a nonzero element in the square of the maximal ideal (provided the depth of the ring is large enough).

Proof of (3.1). Since $M$ lifts to $Q$, by (1.1), there exists an acyclic lifting $(\mathbf{L}, d)$ of $(\mathbf{F}, \partial)$ to $Q$. Let $f: \mathbf{L} \otimes_{Q} R \rightarrow \mathbf{F}$ be a chain isomorphism. Choose liftings $\tilde{f}_{i}: L_{i} \rightarrow Q^{b_{i}}$ of each of the isomorphisms $f_{i}: L_{i} \otimes_{Q} R \rightarrow R^{b_{i}}$, and liftings $\partial_{i}^{\prime}$ to $Q$ of each matrix $\partial_{i}$. Note that each $\tilde{f}_{i}$ is an isomorphism. For all $i$ consider the diagram

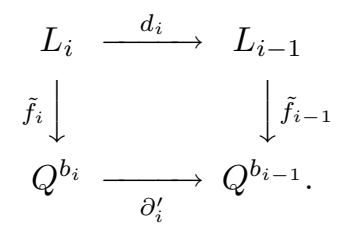

We have $\tilde{f}_{i-1} d_{i}-\partial_{i}^{\prime} \tilde{f}_{i} \equiv 0 \bmod I$. Therefore, thinking of the map $\left(\tilde{f}_{i-1} d_{i}-\right.$ $\left.\partial_{i}^{\prime} \tilde{f}_{i}\right) \tilde{f}_{i}^{-1}: Q^{b_{i}} \rightarrow Q^{b_{i-1}}$ as a matrix, we see that each of its entries is an element of $I$. Thus the matrix $\tilde{\partial}_{i}:=\partial_{i}^{\prime}+\left(\tilde{f}_{i-1} d_{i}-\partial_{i}^{\prime} \tilde{f}_{i}\right) \tilde{f}_{i}^{-1}$ is a lifting to $Q$ of the matrix $\partial_{i}$ which, in place of $\partial_{i}^{\prime}$, makes the diagram above commute. As this holds for all $i$, we see that the resulting sequence

$$
(\widetilde{\mathbf{F}}, \tilde{\partial}): \quad \cdots \rightarrow Q^{b_{i+1}} \stackrel{\tilde{\partial}_{i+1}}{\longrightarrow} Q^{b_{i}} \stackrel{\tilde{\partial}_{i}}{\longrightarrow} \cdots \stackrel{\tilde{\partial}_{2}}{\longrightarrow} Q^{b_{1}} \stackrel{\tilde{\partial}_{1}}{\longrightarrow} Q^{b_{0}} \rightarrow 0
$$

is an acyclic complex.

\section{REFERENCES}

[B] W. Bruns, "Jede" endliche freie Auflösung ist freie Auflösung eines von drei Elementen erzeugten Ideals, J. Algebra 39 (1976), 429 - 439. MR 53:2925

[BE1] D. Buchsbaum and D. Eisenbud, Some structure theorems for finite free resolutions, Advances in math. 12 (1974), 84-139. MR 49:4995

[BE2] L Lifting modules and a theorem on finite free resolutions, Ring Theory (Robert Gordon, ed.), Academic Press, New York, London, 1972, pp. 63-74. MR 49:5098

[BH] W. Bruns and J. Herzog, Cohen-Macaulay Rings, Cambridge University Press, Cambridge, 1993. MR 95h: 13020

[H] M. Hochster, An obstruction to lifting cyclic modules, Pacific J. Math. 61 (1975), 457 463. MR 54:296

[Ho] J. W. Hoffman, Counterexamples to the lifting problem for singularities, Comm. Algebra 11 (1983), 523 - 549. MR 84g:13027

[Mac] D. Bayer and M. Stillman, Macaulay, a computer algebra system for computing in algebraic geometry and commutative algebra, 1990.

[PS] C. Peskine and L. Szpiro, Dimension projective finie et cohomologie locale, Publ. Math. I.H.E.S. Paris, 42 (1973), 323-395. MR 51:10330

Department of Mathematics, University of Texas, Austin, Texas 78712

Current address: Department of Mathematics, University of Texas at Arlington, Arlington, Texas 76019

E-mail address: djorgens@math.uta.edu 